\title{
Power Flow Analysis of Weakly Meshed Distribution Network Including DG
}

\author{
Abdulrahman Al-Sakkaf \\ King Fahd University of Petroleum and Minerals \\ Dhahran, Saudi Arabia
}

\author{
Mohammad AlMuhaini \\ King Fahd University of Petroleum and Minerals \\ Dhahran, Saudi Arabia
}

\begin{abstract}
Power flow is one of the essential studies in power system operation and planning. All steady-state parameters for power distribution systems, such as bus voltage magnitudes, angles, power flows, and power losses, can be calculated by conducting power flow analysis. Distribution system features differ from those of transmission system, rendering conventional load flow algorithms inapplicable. In this paper, three distribution power flow techniques are presented and tested to evaluate their performance when applied to a networked distribution system including distributed generation (DG). These are the distribution load flow (DLF) matrix, the enhanced Newton Raphson (ENR), and the robust decoupled (RD) method. IEEE 33-bus system is adopted for implementing the above methods. Radial and weakly meshed configurations are applied to the tested system with DG inclusion to investigate their influence on the power flow study findings.
\end{abstract}

Keywords-power flow analysis; distribution systems; load flow algorithms; weakly meshed configuration; distributed generator

\section{INTRODUCTION}

Power flow analysis, also known as "load flow" analysis, is one of the most important power system techniques. This analysis can be performed to determine the steady state conditions of a power system for a set of specified power generations (DGs) and load demand conditions. Power flow analysis is essential for power planning and operation, economic scheduling, and power exchange among utilities. Furthermore, it is necessary for other analyses, such as fault analysis, stability, and power system reliability. Power systems consist of three main parts: generation, transmission, and distribution system. Since the distribution system is the final stage in the power system and is connected directly to the customers, its voltage profile must be investigated and evaluated to ensure high-quality service and identify any improvement needs. In order to improve the distribution network performance, DGs, management technologies, power electronic interfaces, and control systems are integrated into the system. This integrated system is called a "microgrid" or "active distribution network". To study the voltage profile of an active distribution or microgrid system, power flow analysis must be performed.

Power flow calculation is usually carried out using the node voltage method. First, an admittance matrix for the system is formed, before deriving complex nonlinear power flow equations. Finally, node voltages and other parameters are calculated by solving the power flow equations. The most common techniques used for load flow calculation are GaussSeidel, Newton-Raphson, and fast decoupled iterative techniques $[1,2]$. These methods were developed to solve the power flow for transmission systems. However, they may not work appropriately in distribution systems, as they exhibit the following special features [3]:

- Radial and sometimes weakly meshed structures

- High resistance to reactance (r/x) ratios

- Multiphase and unbalanced orientation

- Extremely large number of branches and nodes

For a distribution system, techniques based on nodes and branches have been developed and are widely used. One of the most common methods is the forward and backward sweep method [4]. This method can be used to solve power flow equations for weakly meshed distribution systems by converting the weakly meshed into a radial system and solving it directly by Kirchhoff's voltage and current laws (KVL and $\mathrm{KCL}$ ). In this case, the number of iterations is high due to the conversion process. A fast decoupled method using G-matrix (FDG) for application in distribution systems has been outlined in [5]. This method relies on the Newton-Raphson technique with rectangular coordinates and equivalent current injections. The FDG method is sensitive to the reactance $(\mathrm{r} / \mathrm{x})$ ratio. Simple and efficient branch-to-node matrix-based power flow (BNPF) for radial distribution systems has been presented in [6]. A key feature of this method is that voltages for all nodes are calculated from the source voltage. A branch-to-node matrix is used to define the relationship between the branch current and node currents without the need for matrix inversion. A distribution power flow technique aiming to enhance the convergence rate by partially linearizing the power flow equations has been developed in [7]. This method is insensitive to the resistance to the $\mathrm{r} / \mathrm{x}$ ratio and is suitable for large-scale distribution systems. On the other hand, forming, calculating, and inverting the Jacobian matrix increases its complexity, making it more time-consuming.

In this paper, three distribution power flow techniques will be presented and tested on an IEEE 33-bus system. These are the distribution load flow (DLF) matrix, the enhanced Newton 
Raphson (ENR), and the robust decoupled (RD) method. Radial and weakly meshed configuration will be implemented to evaluate the effect of the network structure on the power flow study. In addition, distributed generation (DG) will be included in order to study its impact on the distribution system voltage profile.

\section{POWER FLOW TECHNIQUES}

\section{A. Distribution Load Flow (DLF) Method}

This method solves the distribution power flow directly by using two matrices: bus injection to branch current (BIBC) and branch current to bus voltage (BCBV) matrix [8]. BIBC and $\mathrm{BCBV}$ are formed based on the system configuration and are utilized for branch current and voltage drop calculations, respectively. Multiplying those two matrices results in the distribution load flow (DLF) matrix, which is used in one step to compute bus voltages. The power distribution system shown in Figure 1 is used as an example. At the beginning, we assume that all buses or node voltages are equal to that at the source node. Specified data in the distribution system are load active power $(P)$ and reactive power $(Q)$ for each bus. Node currents can be computed in terms of specified $P$ and $Q$ using (1):

$$
I_{\text {node }}=\frac{(P+Q)^{*}}{V^{*}}
$$

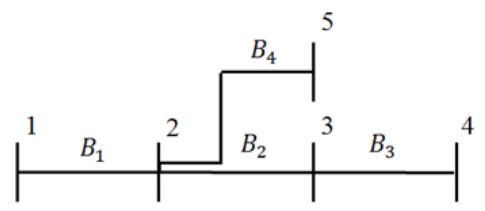

Fig. 1. Sample distribution system

By applying KCL to the distribution system, branch currents can be obtained in terms of node currents by applying the following equations:

$$
\begin{aligned}
& I_{\text {branch } 1}=I_{\text {node } 2}+I_{\text {node } 3}+I_{\text {node } 4}+I_{\text {node } 5} \\
& I_{\text {branch } 2}=I_{\text {node } 3}+I_{\text {node } 4} \\
& I_{\text {branch } 3}=I_{\text {node } 4} \\
& I_{\text {branch } 4}=I_{\text {node } 5}
\end{aligned}
$$

The relationship between branch currents and node currents in (2) can be expressed via the BIBC matrix as follows:

$$
\left[\begin{array}{l}
I_{\text {branch } 1} \\
I_{\text {branch } 2} \\
I_{\text {branch } 3} \\
I_{\text {branch4 }}
\end{array}\right]=\left[\begin{array}{llll}
1 & 1 & 1 & 1 \\
0 & 1 & 1 & 0 \\
0 & 0 & 1 & 0 \\
0 & 0 & 0 & 1
\end{array}\right]\left[\begin{array}{l}
I_{\text {node2 }} \\
I_{\text {node3 }} \\
I_{\text {node } 4} \\
I_{\text {node } 5}
\end{array}\right]
$$

Equation (3) can be expressed as:

$$
I_{\text {branch }}=[B I B C] * I_{\text {node }}
$$

Bus voltages can be calculated in terms of drop voltages by applying the following equalities:

$$
\begin{aligned}
& V_{2}=V_{1}-\left(I_{\text {branch } 1} * Z_{B 1}\right)=V_{1}-V_{D 1} \\
& V_{3}=V_{2}-\left(I_{\text {branch } 2} * Z_{B 2}\right)=V_{2}-V_{D 2}
\end{aligned}
$$

$$
\begin{aligned}
& V_{4}=V_{3}-\left(I_{\text {branch } 3} * Z_{B 3}\right)=V_{3}-V_{D 3} \\
& V_{5}=V_{2}-\left(I_{\text {branch } 4} * Z_{B 4}\right)=V_{2}-V_{D 4}
\end{aligned}
$$

The relationship between branch currents and bus voltages in (5) can be expressed via the BCBV matrix as follows:

$$
\left[\begin{array}{l}
V_{D 1} \\
V_{D 2} \\
V_{D 3} \\
V_{D 4}
\end{array}\right]=\left[\begin{array}{cccc}
Z_{B 1} & 0 & 0 & 0 \\
Z_{B 1} & Z_{B 2} & 0 & 0 \\
Z_{B 1} & Z_{B 2} & Z_{B 3} & 0 \\
Z_{B 1} & 0 & 0 & Z_{B 4}
\end{array}\right]\left[\begin{array}{c}
I_{\text {branch } 1} \\
I_{\text {branch } 2} \\
I_{\text {branch } 3} \\
I_{\text {branch } 4}
\end{array}\right]
$$
obtain:

By rewriting (6) in the general expression format, we

$$
V_{D}=[B C B V] * I_{\text {branch }}
$$

Substituting (4) in (7) yields:

$$
\begin{aligned}
V_{D} & =[B C B V] *[B I B C] * I_{\text {node }} \\
V_{D} & =[D L F] * I_{\text {node }}
\end{aligned}
$$

where DLF is the distribution load flow matrix:

$$
D L F=\left[\begin{array}{cccr}
Z_{B 1} & Z_{B 1} & Z_{B 1} & Z_{B 1} \\
Z_{B 1} & Z_{B 1}+Z_{B 2} & Z_{B 1}+Z_{B 2} & Z_{B 1} \\
Z_{B 1} & Z_{B 1}+Z_{B 2} & Z_{B 1}+Z_{B 2}+Z_{B 3} & Z_{B 1} \\
Z_{B 1} & Z_{B 1} & Z_{B 1} & Z_{B 1}+Z_{B 4}
\end{array}\right]
$$

Finally, bus voltages are computed as:

$$
V_{\text {Bus }}=V_{\text {Source }}-V_{\text {drop }}
$$

After solving the voltages for all buses by applying (9), we need to check for convergence. If the solution has converged, the process is terminated. Otherwise, the algorithm is executed iteratively until the convergence criteria are met. Figure 2 shows the algorithm of the DLF method.

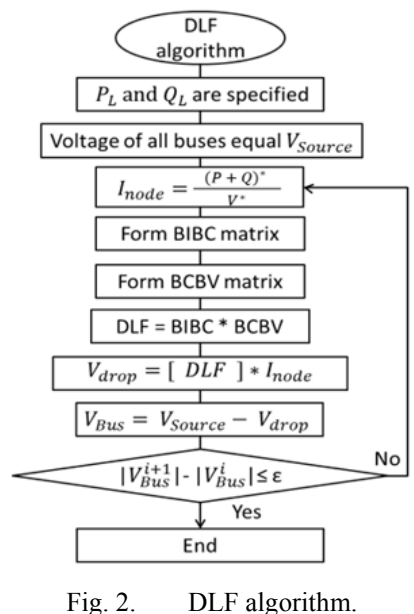

\section{B. Enhanced Newton Raphson (ENR) Method}

This method provides a solution for distribution load flow using the current-injection technique based on the NewtonRapson method and rectangular coordinates [5]. The Jacobian matrix in ENR is called "G matrix." Unlike conventional Newton Raphson method, the matrix is formed only once and remains unchanged. For the first time, decoupling can be applied to the distribution power flow by assuming off- 
diagonal blocks of the $\mathrm{G}$ matrix to be zeros. For a distribution system with $n$ buses, the specified node current can be calculated in terms of specified active power $(P)$ and reactive power $(Q)$ as follows:

$$
I_{\text {node }}=\frac{(P+Q)^{*}}{V^{*}}
$$

Specified branch currents can be calculated as:

$$
I_{\text {branch }}^{\text {sp }}=[C] * I_{\text {node }}
$$

where $C$ is a branch to bus matrix. Bus voltage will be represented in rectangular coordinates as:

$$
V=e+j f
$$

By substituting (10) and (12) in (11), equation (11) becomes:

$$
I_{\text {branch }}^{s p}=[C] * \frac{(P+Q)^{*}}{(e+j f)^{*}}
$$

Branch current can also be calculated in terms of branch admittance in rectangular coordinates:

$$
I_{\text {branch }}=\left[\left(e_{i}-e_{i+1}\right)+j\left(f_{i}-f_{i+1}\right)\right]\left[G_{j}-B_{j}\right]
$$

where $i$ represents a particular bus in the system, where $i=1,2$, $3, \ldots, n$, and $j$ denotes the branches $(j=1,2,3, \ldots, n-1)$. By separating the real and imaginary parts of (13) and (14), the Newton-Raphson solution for the load flow can be formulated as:

$$
\left[\begin{array}{ll}
J_{1} & J_{2} \\
J_{3} & J_{4}
\end{array}\right]\left[\begin{array}{l}
\Delta e \\
\Delta f
\end{array}\right]=\left[\begin{array}{l}
\Delta I_{\text {branch }}^{r} \\
\Delta I_{\text {branch }}^{i}
\end{array}\right]
$$

Jacobians are calculated only once, as follows:

$$
\begin{aligned}
& J_{1}=\frac{\partial I_{\text {branch }}^{r}(e, f)}{\partial e}=G, J_{2}=\frac{\partial I_{\text {branch }}^{r}(e, f)}{\partial f}=-B \\
& J_{3}=\frac{\partial I_{\text {branch }}^{i}(e, f)}{\partial e}=G, J_{4}=\frac{\partial I_{\text {branch }}^{i}(e, f)}{\partial f}=B
\end{aligned}
$$

Current mismatches $\Delta I_{\text {branch }}^{r}$ and $\Delta I_{\text {branch }}^{i}$ are calculated using the expressions below:

$$
\begin{aligned}
& \Delta I_{\text {branch }}^{r}=\operatorname{Re}\left(I_{\text {branch }}^{\text {sp }}\right)-\operatorname{Re}\left(I_{\text {branch }}\right) \\
& \Delta I_{\text {branch }}^{i}=\operatorname{Im}\left(I_{\text {branch }}^{\text {sp }}\right)-\operatorname{Im}\left(I_{\text {branch }}\right)
\end{aligned}
$$
(15):

Correction values $\Delta e$ and $\Delta f$ can be obtained by factorizing

$$
\left[\begin{array}{l}
\Delta e \\
\Delta f
\end{array}\right]=\left[\begin{array}{cc}
G & -B \\
B & G
\end{array}\right]^{-1}\left[\begin{array}{l}
\Delta I_{\text {branch }}^{r} \\
\Delta I_{\text {branch }}^{i}
\end{array}\right]
$$

Finally, voltage magnitudes and angles are computed and updated by:

$$
\begin{aligned}
& e_{\text {new }}=e_{\text {old }}+\Delta e \\
& f_{\text {new }}=f_{\text {old }}+\Delta f
\end{aligned}
$$

After computing $e$ and $f$ by applying (18), we need to check for convergence. If the solution has converged, the process is terminated. Otherwise, the algorithm is executed iteratively until the convergence criteria are met. Figure 3 shows the algorithm of the ENR method. The G matrix can be decoupled by assuming that the off-diagonal blocks of $G$ matrix have zero value.
$\left[\begin{array}{l}\Delta e \\ \Delta f\end{array}\right]=\left[\begin{array}{ll}G & 0 \\ 0 & G\end{array}\right]^{-1}\left[\begin{array}{l}\Delta I_{\text {branch }}^{r} \\ \Delta I_{\text {branch }}^{i}\end{array}\right]$

Consequently, $\Delta e$ and $\Delta f$ can be calculated as:

$$
\begin{aligned}
& \Delta e=G \Delta I_{\text {branch }}^{r} \\
& \Delta f=G \Delta I_{\text {branch }}^{i}
\end{aligned}
$$

A drawback of this assumption is that, in large systems, the solution may not converge.

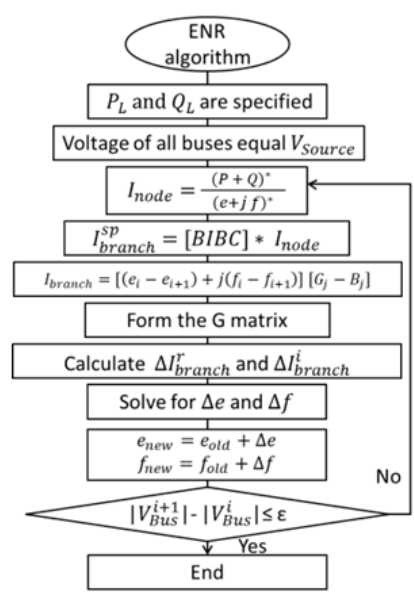

Fig. 3. ENR algorithm.

\section{Robust Decoupled (RD) Method}

The main objective of the robust decoupled method is to decouple the Newton-Raphson equation into two sub-equations by a constant transformation matrix [9]. Multiplying the Jacobian matrix by the transformation matrix will result in zero off-diagonal blocks of the Jacobian. Equation (15) is multiplied by the transformation matrix in order to decouple the equation.

$$
\left[\begin{array}{cc}
T_{1} & T_{2} \\
-T_{2} & T_{1}
\end{array}\right]\left[\begin{array}{ll}
J_{1} & J_{2} \\
J_{3} & J_{4}
\end{array}\right]\left[\begin{array}{l}
\Delta e \\
\Delta f
\end{array}\right]=\left[\begin{array}{cc}
T_{1} & T_{2} \\
-T_{2} & T_{1}
\end{array}\right]\left[\begin{array}{l}
\Delta I_{\text {branch }}^{r} \\
\Delta I_{\text {branch }}^{i}
\end{array}\right]
$$

$T_{1}$ and $T_{2}$ are diagonal matrices of cosine and sine admittance angles, respectively. After decoupling, (21) will be represented as:

$$
\begin{aligned}
& {[\mathrm{T}][\Delta \mathrm{e}]=\left[\Delta \mathrm{I}_{\text {branch }}^{\mathrm{r}-\mathrm{T}}\right]} \\
& {[\mathrm{T}][\Delta \mathrm{f}]=\left[\Delta \mathrm{I}_{\text {branch }}^{\mathrm{i}}\right]}
\end{aligned}
$$

Sub-Jacobian matrix $\mathrm{T}, \Delta I_{\text {branch }}^{r_{-} T}$, and $\Delta I_{\text {branch }}^{i_{-} T}$ are calculated using the expressions below:

$$
\begin{aligned}
& {[T]=\left[T_{1}\right] \frac{\partial I_{\text {branch }}^{r}(e, f)}{\partial e}+\left[T_{2}\right] \frac{\partial I_{\text {branch }}^{i}(e, f)}{\partial e}} \\
& =\left[-T_{2}\right] \frac{\partial I_{\text {branch }}^{r}(e, f)}{\partial f}+\left[T_{1}\right] \frac{\partial I_{\text {branch }}^{i}(e, f)}{\partial f} \\
& {\left[\Delta I_{\text {branch }}^{r_{T}}\right]=\left[T_{1}\right]\left[\Delta I_{\text {branch }}^{r}\right]+\left[T_{2}\right]\left[\Delta I_{\text {branch }}^{i}\right]} \\
& {\left[\Delta I_{\text {branch }}^{i_{T}}\right]=\left[-T_{2}\right]\left[\Delta I_{\text {branch }}^{r}\right]+\left[T_{1}\right]\left[\Delta I_{\text {branch }}^{i}\right]}
\end{aligned}
$$


Equation (23) is calculated only once as the Jacobian. Correction values $\Delta e$ and $\Delta f$ can be obtained by dividing (22) by $[T]$ :

$$
\begin{aligned}
& {[\Delta e]=[T]^{-1}\left[\Delta I_{\text {branch }}^{r_{-} T}\right]} \\
& {[\Delta \mathrm{f}]=[\mathrm{T}]^{-1}\left[\Delta \mathrm{I}_{\text {branch }}^{\mathrm{i} \mathrm{T}}\right]}
\end{aligned}
$$

Finally, voltage magnitudes and angles are computed by:

$$
\begin{aligned}
& e_{\text {new }}=e_{\text {old }}+\Delta e \\
& f_{\text {new }}=f_{\text {old }}+\Delta f
\end{aligned}
$$

After computing $e$ and $f$ by applying (26), the voltage magnitudes and angles are updated until the convergence criteria are met, as shown in Figure 4.

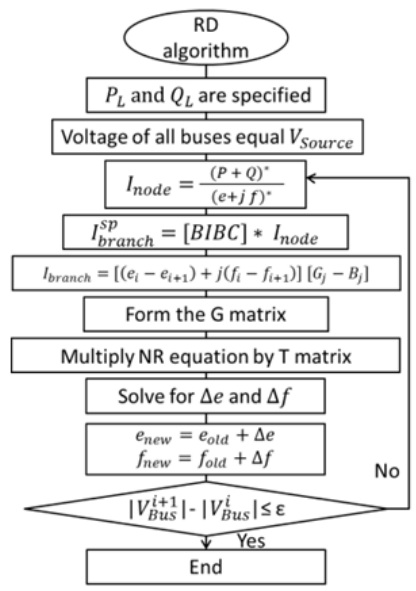

Fig. 4. RD algorithm.

\section{IMPACTS OF WEAKLY MESHED CONNECTION \& DG ON POWER FLOW TECHNIQUES}

\section{A. Weakly Meshed Distribution Load Flow}

A distribution system typically comprises of a large number of feeders and load points connected to these feeders, as well as open tie switches. Tie switches are closed in contingency or when serving high-density loads. When some of the tie switches are closed, this creates loops in the distribution system, resulting in a weakly meshed rather than radial configuration. This new configuration leads to loss reduction, voltage profile improvement, as well as reliability and powerquality enhancement. A simple weakly meshed distribution system is shown in Figure 5.

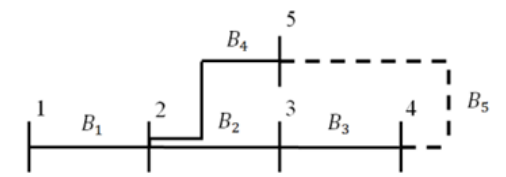

Fig. 5. A weakly meshed distribution system

DLF method can be utilized in this case with few modifications. As shown in Figure 5, a new branch $\left(B_{5}\right)$ is added to the system. The branch current $I_{\text {branch } 5}$ flows through
$B_{5}$ from node 4 to node $5 . I_{\text {node } 4}$ and $I_{\text {node } 5}$ will be calculated as [10]:

$$
\begin{aligned}
& I_{\text {node4 }}^{\prime}=I_{\text {node } 4}+I_{\text {branch } 5} \\
& I_{\text {node5 }}^{\prime}=I_{\text {node5 } 5}-I_{\text {branch } 5}
\end{aligned}
$$

DLF matrix will be modified as well. If a new branch is added from a bus $i$ to a bus $j$, all elements in the $j_{t h}$ column are subtracted from the corresponding elements in the $i_{t h}$ column in the existing DLF matrix and the results represent the new $k_{t h}$ column and $k_{t h}$ row of the DLF matrix. The diagonal element of the $k_{t h}$ row and column is found by summing all the impedances of the branches involved in forming the loop or "weak mesh."

$$
D L F=\left[\begin{array}{ccccc}
Z_{11} & Z_{12} & Z_{13} & Z_{14} & Z_{13-14} \\
Z_{21} & Z_{22} & Z_{23} & Z_{24} & Z_{23-24} \\
Z_{31} & Z_{32} & Z_{33} & Z_{34} & Z_{33-34} \\
Z_{41} & Z_{42} & Z_{43} & Z_{44} & Z_{43-44} \\
Z_{13-14} & Z_{23-24} & Z_{33-34} & Z_{43-44} & Z_{55}
\end{array}\right]
$$

After modifying the DLF matrix, Kron's reduction must be applied to the new DLF matrix to ensure that it is of the same dimensions as before [1]:

$$
Z_{\text {new }}=Z_{\text {old }}-\frac{\text { corresponding }\left(Z_{i-j(\text { column })} * Z_{i-j(\text { row })}\right)}{Z_{i-j(\text { diagonal })}}
$$

This technique is applicable to the DLF method only, as it requires DLF matrix modification.

\section{B. Distribution Load Flow Including $D G$}

Distribution generation (DG) is a generation system that spreads through the distribution system for the purpose of improving service reliability. DG could be a photovoltaic system, a wind turbine, a fuel cell, a micro turbine, etc. These DG units have different operational modes [11]. They could supply a power with a predetermined amount of reactive power (fixed power factor). In this case, DG units are not controlling the voltage at the connection point. On the other hand, DG units could supply power by controlling and regulating the voltage at the connection points.

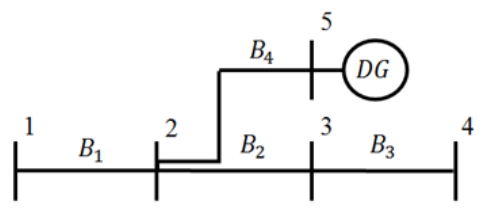

Fig. 6. A distribution system with distributed generator

If a DG unit generates a predetermined amount of reactive power, the DG is modelled as a PQ bus and is treated as a load bus with negative $\mathrm{P}$ and $\mathrm{Q}$ values. However, when a DG unit regulates and controls the voltage at the connection point, the DG is modelled as a PV bus. In the case of PV, active DG power $P_{D G}$ is specified, reactive DG power $Q_{D G}$ is initially set to zero, and the voltage at the $\mathrm{P}-\mathrm{V}$ node is set to the desired value. The DLF, ENR, or RD method can be utilized here with some adjustments. Branch currents will be calculated as:

$$
I_{\text {branch }}=[B I B C] *\left(I_{\text {node }}+I_{D G}\right)
$$


After computing the bus voltages, if $\left|V_{i}-V_{i \text { set }}\right| \leq$ error tolerance, the solution has converged. Otherwise, $Q_{D G}$ will be generated by the PV node to maintain the voltage at the specified value. $Q_{D G}$ can be computed as:

$$
Q_{D G \text { new }}=Q_{D G \text { old }}+\operatorname{Im}\left(V_{i} \frac{V_{i}^{*}}{Z}\right)
$$

where $i$ is the number of the DG bus, $V_{i \text { set }}$ is the set voltage at the DG bus, and $V_{i}$ is the calculated voltage at the DG bus. The algorithm for solving distribution power flow with DG inclusion is the same as the previously described method based on those two equations.

\section{TESTS AND RESULTS}

Tests and simulations for the presented methods were carried out. DLF, ENR, and RD, were tested on a radial 33-bus distribution system. In addition, the DLF method was tested on a weakly meshed 33-bus distribution system. Moreover, the DLF method was tested on a weakly meshed 33-bus distribution system including DG. Line and load data for the system are based on [12]. The base data is $12.66 \mathrm{kV}$ and $10 \mathrm{MW}$, and the convergence tolerance is 0.0001 .

\section{A. Radial Distribution System}

The previously described distribution load flow methods, DLF, ENR, and RD, were tested on a radial 33-bus [12] in order to assess their performance, accuracy, speed of calculation, robustness, and computational efficiency. Figure 7 shows the IEEE radial 33-bus system. Solutions for DLF, ENR, and $\mathrm{RD}$ for the radial 33-bus are shown in Table I.

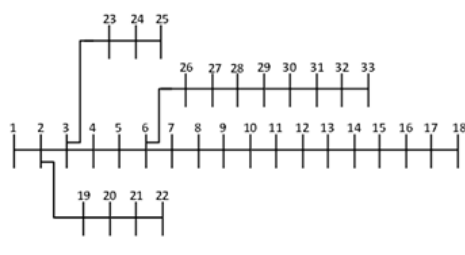

Fig. 7. 33-bus distribution system.

TABLE I. PF SOLUTION FOR RADIAL 33-BUS SYSTEM

\begin{tabular}{|c|c|c|c|c|c|}
\hline \multirow{2}{*}{ Bus no. } & \multicolumn{2}{|c|}{ DLF, ENR, and RD } & \multirow{2}{*}{ Bus no. } & \multicolumn{2}{c|}{ DLF, ENR, and RD } \\
\cline { 2 - 3 } & $\boldsymbol{V}_{\boldsymbol{p u}}$ & $\boldsymbol{\delta}_{\boldsymbol{d e g}}$ & & $\boldsymbol{V}_{\boldsymbol{p} \boldsymbol{u}}$ & $\boldsymbol{\delta}_{\boldsymbol{d e g}}$ \\
\hline 1 & 1 & 0 & 18 & 0.9131 & -0.4950 \\
\hline 2 & 0.9970 & 0.0145 & 19 & 0.9965 & 0.0037 \\
\hline 3 & 0.9829 & 0.0960 & 20 & 0.9929 & -0.0633 \\
\hline 4 & 0.9755 & 0.1616 & 21 & 0.9922 & -0.0827 \\
\hline 5 & 0.9681 & 0.2283 & 22 & 0.9916 & -0.1030 \\
\hline 6 & 0.9497 & 0.1338 & 23 & 0.9794 & 0.0651 \\
\hline 7 & 0.9462 & -0.0965 & 24 & 0.9727 & -0.0237 \\
\hline 8 & 0.9413 & -0.0604 & 25 & 0.9694 & -0.0674 \\
\hline 9 & 0.9351 & -0.1335 & 26 & 0.9477 & 0.1733 \\
\hline 10 & 0.9292 & -0.1960 & 27 & 0.9452 & 0.2295 \\
\hline 11 & 0.9284 & -0.1888 & 28 & 0.9337 & 0.3124 \\
\hline 12 & 0.9269 & -0.1773 & 29 & 0.9255 & 0.3903 \\
\hline 13 & 0.9208 & -0.2686 & 30 & 0.9220 & 0.4956 \\
\hline 14 & 0.9185 & -0.3473 & 31 & 0.9178 & 0.4112 \\
\hline 15 & 0.9171 & -0.3849 & 32 & 0.9169 & 0.3881 \\
\hline 16 & 0.9157 & -0.4082 & 33 & 0.9166 & 0.3804 \\
\hline 17 & 0.9137 & -0.4855 & & & \\
\hline
\end{tabular}

Results reported in Table I indicate that all three methods provide the same solution, which is consistent with those reported in $[13,14]$. For DLF, ENR, and RD, the solution converged after the same number of iterations (4), thus, all three methods have good convergence. In terms of computational time, DLF outperforms ENR and RD, as $46 \mathrm{~ms}$, $70 \mathrm{~ms}$, and $80 \mathrm{~ms}$ were obtained, respectively. Nonetheless, all three methods are accurate and efficient. However, DLF is simpler and faster than ENR and RD. Table II shows the comparison among these three methods.

TABLE II. COMPARISON OF THE PROPOSED METHODS

\begin{tabular}{|c|c|c|c|}
\hline DPF methods & DLF & ENR & RD \\
\hline Accuracy & accurate & accurate & accurate \\
\hline Number of iterations & 4 & 4 & 4 \\
\hline Computational time & $46 \mathrm{~ms}$ & $70 \mathrm{~ms}$ & $80 \mathrm{~ms}$ \\
\hline Flexibility & flexible & inflexible & inflexible \\
\hline Complexity & simple & complex & complex \\
\hline Sensitivity to r/x ratio & insensitive & insensitive & insensitive \\
\hline
\end{tabular}

\section{B. Weakly Meshed Distribution System}

DLF method with the adopted modification for a weakly meshed distribution system was tested on the 33-bus system having five loops [10], as shown in Figure 8. The new mesh connections cause a reduction in the branch current values. This reduces drop voltage and power losses, while improving the voltage profile. The results obtained from the power flow solution are identical to the results reported in [10]. The voltage profile is improved due to closing the tie lines and all bus voltages, which are thus within $\pm 5 \%$ of the base voltage, as shown in Figure 12. The improvements range from $-1.68 \%$ to $+4.44 \%$. Although voltages at bus 19-22 and 24-25 decrease slightly, they remain within the acceptable voltage limits. Figure 9 shows the percentage improvements in the voltage profile due to the mesh connections. Red points on the graph indicate voltage deterioration.

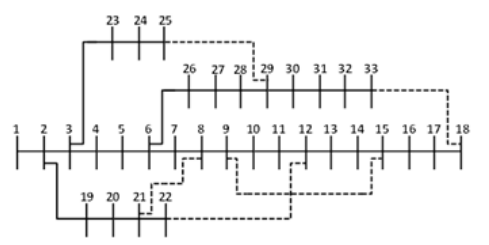

Fig. 8. 33-bus distribution system with 5 closed tie lines

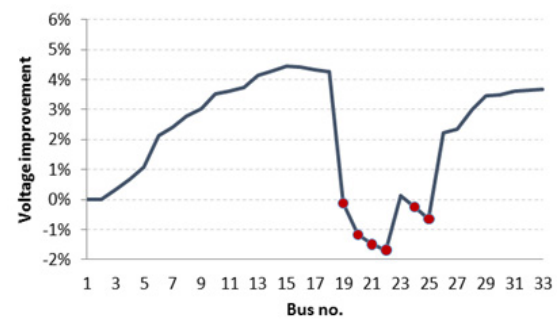

Fig. 9. Voltage profile improvements due to mesh connections.

\section{Weakly Meshed Distribution System with DG}

The DLF method with the additional equations required for including the DG unit in the distribution system was tested on the 33-bus weakly meshed distribution system. Figure 10 shows that DG generating $1000 \mathrm{~kW}$ was installed at bus 22 . The 
DG is modelled as a PV bus controlling the voltage at the connection point. The voltage at bus 22 is set at 1p.u. The voltage profile is improved because the weakly meshed and distributed generator is connected to the system, where DG generates $970 \mathrm{kvar}$. This improvement extends from $-0.1 \%$ to $+5.80 \%$, whereby only one bus voltage decreases by $0.1 \%$ (at bus 25), as shown in Figure 11.

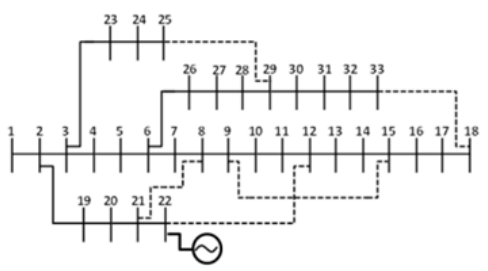

Fig. 10. A 33-bus weakly meshed distribution system with DG.

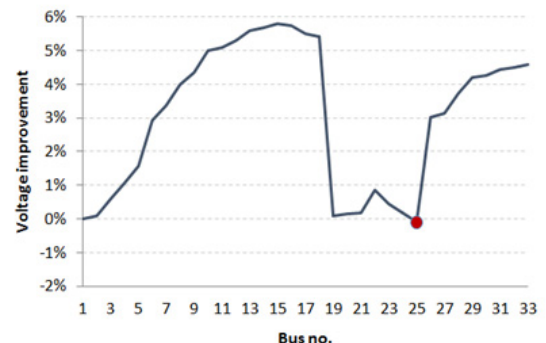

Fig. 11. Voltage profile improvement due to $1000 \mathrm{~kW}$ DG.

An illustration of the voltage profile for the three aforementioned test systems is shown in Figure 12. In the radial system, voltage violations occurred, and several buses had a voltage below 0.95 p.u., as indicated by red dots. The weakly meshed connection improved the voltage profile and eliminated violations. Moreover, all bus voltages were within the 1.05 p.u. -0.95 p.u. range. Summarizing, installing DG at bus 22 in the weakly meshed system enhanced further the system voltage.

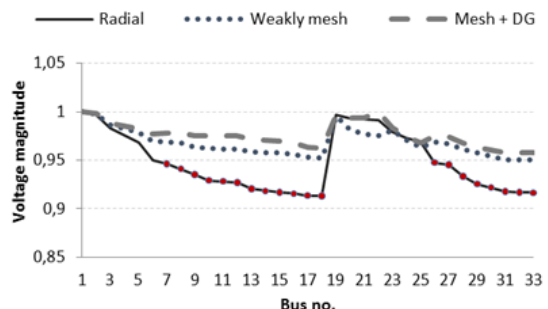

Fig. 12. Voltage profiles of the radial, weakly meshed, and weakly meshed system with DG.

\section{1) Installing Different Sizes of $D G$}

A variety of DG sizes were tested to investigate the effects of DG size on the power flow study and voltage profile. Three DGs $(200 \mathrm{~kW}, 800 \mathrm{~kW}$, and $1500 \mathrm{~kW}$ respectively) were installed at bus 22, one at the time. Error tolerance was reduced to 0.001 to ensure better convergence. Figures 13 and 14 show maximum improvement and deterioration in the system voltage for each DG size. As previously noted, the deterioration occurred at bus 25 only. Average voltage enhancements for the system with the DG of $200 \mathrm{~kW}, 800 \mathrm{~kW}$, and $1500 \mathrm{~kW}$ size are computed as $3.05 \%, 3.13 \%$, and $3.20 \%$, respectively. Thus, the voltage profile is slightly enhanced with the increment in the DG size.

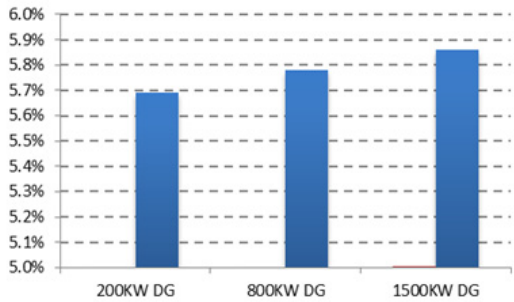

Fig. 13. Maximum voltage improvement due to different DG sizes.

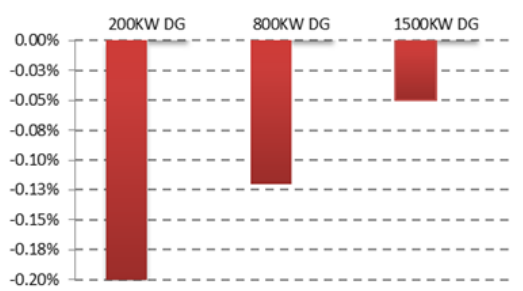

Fig. 14. Maximum voltage deterioration due to different DG sizes.

\section{2) Installing a DG at Different Locations}

In addition to investigating the effects of different DG sizes, a $1000 \mathrm{~kW}$ DG was installed at different locations in the system, and tests were performed to study the DG location influence on the system voltage. The DG was installed at bus 6 , 18,22 , and 33, respectively, one at a time. Power flow results for each case are shown in Figure 15. The reactive powers required to obtain the power flow solution for the system with DG at bus 6, 18, 22, and 33 are 3485 kvar, 1047 kvar, 970 kvar, and $1540 \mathrm{kvar}$, respectively.

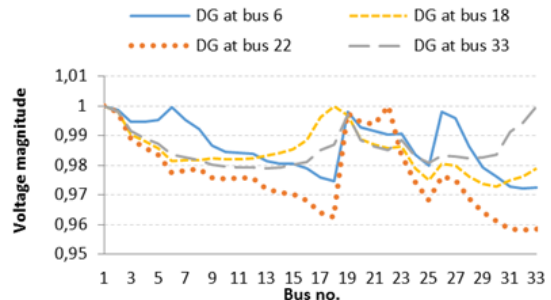

Fig. 15. Voltage profiles of the weakly meshed system with DG at different locations.

\section{3) Installing Multiple DGs}

In this case, three DGs were installed at the same time in different locations to study the impact of multiple DGs on the system. Two DGs were modeled as PV and were installed at bus 18 and 22, respectively, while the third DG was modeled as PQ and was installed at bus 33. The two PV DGs produced $1000 \mathrm{~kW}$ and were set at 1p.u. voltage, whereas the PQ DG generated $500 \mathrm{~kW}$ and $500 \mathrm{kvar}$. Measured improvements range from $+0.18 \%$ to $+9.53 \%$, as shown in Figure 16. In this case, all bus voltages are improved and none exhibits any 
deteriorations. These voltage improvements arise as multiple DGs exhibit a greater effect than do individual DGs.

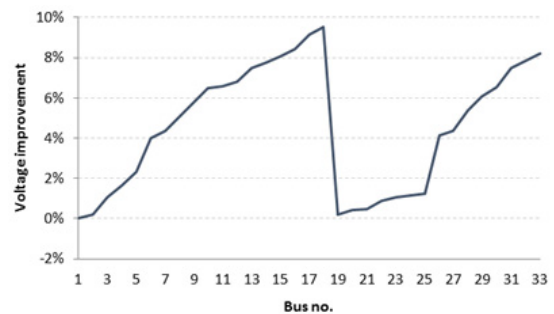

Fig. 16. Voltage profile improvements due to multiple DGs.

\section{Distribution System with Peak Load}

In this scenario, the system loads were increased by $20 \%$ to study the effect of peak loads on the system voltage profile. The IEEE 33-bus distribution system was tested in radial configuration, with weakly meshed connections and multiple DGs. Figure 17 shows the results of the three cases with peak loads. In the radial system, 21 buses have voltages below the limit, as indicated by the red dots on the graph. With the mesh connections, the voltage profile partially improved, as only 10 buses were below the limit. Including multiple DGs improved the voltage profile substantially, eliminating all violations. These results show that increasing the load rate causes a greater number of voltage violations in the system.

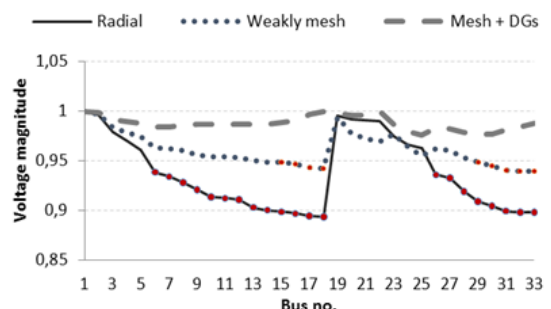

Fig. 17. Voltage profiles of the peak load radial, weakly meshed, and weakly meshed system with DGs.

\section{CONCLUSIONS}

Power flow analysis helps determine the steady state parameters of a power system. Conventional power flow techniques for transmission systems are not applicable to the distribution systems due to their radial nature, extremely large number of components, multiphase operation, and high $\mathrm{r} / \mathrm{x}$ ratios. In this paper, three power flow techniques (DLF, ENR, and RD) suitable for distribution systems were presented. These methods were tested on the IEEE 33-bus radial distribution system. The results showed that all three methods are accurate, have good convergence, are insensitive to $\mathrm{r} / \mathrm{x}$ ratios, and are suitable for large systems. Moreover, DLF was applied to a weakly meshed network including DG to assess its impact on the power flow study. Different tests utilizing DGs were carried out, whereby the size, location, and the number of DGs varied. These three factors play an important role in the effectiveness of utilizing DGs, as they exert significant effects on the distribution system voltage profile. Finally, radial, weakly meshed, and multiple DG tests were repeated with peak load to study the influence of the load rate on the load flow analysis.

\section{REFERENCES}

[1] H. Saadat, Power System Analysis, McGraw Hill, 2004

[2] R. Guguloth, T. K. Sunil Kumar, "Optimal power flow-based congestion management by classical and intelligent methods", International Journal of Electrical Power and Energy Systems, Vol. 36, No. 3, pp. 104-111, 2016

[3] M. S. Srinivas, "Distribution load flows: A brief review", 2000 IEEE Power Engineering Society Winter Meeting, Singapore, Singapore, January 23-27, 2000

[4] D Shirmohammadi, H. W. Hong, A. Semlyen, G. X. Luo, "A compensation-based power flow method for weakly meshed distribution networks", IEEE Transactions on Power Systems, Vol. 3, No. 2, pp. $753-762,1988$

[5] W. M. Lin, J. H. Teng, "Three-phase distribution network fast decoupled power flow solutions", International Journal of Electrical Power and Energy Systems, Vol. 22, No. 5, pp. 375-380, 2000

[6] P. Aravindhababu, S. Ganapathy, K. R. Nayar, "A novel technique for the analysis of radial distribution systems", International Journal of Electrical Power and Energy Systems, Vol. 23, No. 3, pp. 167-171, 2001

[7] R. Ashokkumar, P. Aravindhababu, "An Improved Power Flow Technique for Distribution Systems", Computer Science, Informatics and Electrical Engineering, Vol. 3, No. 1, pp. 1-8, 2009

[8] A. Alsaadi, G. Gholami, "An effective approach for distribution sys-tem for power flow solution", International Journal of Electrical and Computer Engineering, Vol. 3, No. 1, pp. 1-5, 2009

[9] P. Aravindhababu, R. Ashokkumar, "A robust decoupled power flow for distribution systems", Energy Conversion and Management, Vol. 52, No. 4, pp. 1930-1933, 2011

[10] K. Prakash, M. Sydulu, "Topological and Primitive Impedance-Based Load Flow Method for Radial and Weakly Meshed Distribution Systems", Iranian Journal of Electrical and Computer Engineering, Vol. 10, No. 1, pp. 10-18, 2011

[11] H. Shateri, M. Ghorbani, N. Eskandari, A. H. Mohammad-Khani, "Load Flow Method for Unbalanced Distribution Networks with Dispersed Generation Units", 47th International Universities Power Engineering Conference, London, UK, September 4-7, 2012

[12] M. E. Baran, F. F. Wu, "Network reconfiguration in distribution systems for loss reduction and load balancing", IEEE Transactions on Power Delivery, Vol. 4, No. 2, pp. 1401-1407, 1989

[13] J. Z. Zhu, "Optimal reconfiguration of electrical distribution net-work using refined genetic algorithm", Electric Power Systems Research, Vol. 62, pp. 37-42, 2002

[14] M. P. Selvan, K. S. Swarup, "Distribution system load flow using object oriented methodology", 2004 International Conference on Power System Technology, 2004, Singapore, Singapore, November 21-24, 2004 